\title{
A simplified description of bodies floating in the water
}

\author{
Eduardo De Paiva*1] \\ ${ }^{1}$ Instituto de Radioproteção e Dosimetria, Divisão de Física Médica, Rio de Janeiro, RJ, Brasil
}

Received on March 25, 2019; Revised on June 21, 2019; Accepted on June 21, 2019.

\begin{abstract}
The Archimedes' principle and the concepts of equilibrium of forces and density are used to a simple theoretical description of the process of body floating in the water. The analysis is performed for containers easily found in quotidian and is based on the proportion among the air volume inside the container, the water volume within it and on the volume of the container walls. In following, direct experimental demonstrations are proposed.
\end{abstract}

Keywords: Archimedes' principle, buoyancy, force equilibrium, density.

\section{Introduction}

When a body is immersed, even if partially, in a fluid at equilibrium it exerts on the fluid a pressure at all points in contact with it. The fluid reacts (Newton's third law) by exerting a pressure on all points of the surface of the body in contact with it, and this pressure is larger at greater depths. For a vertical cylinder (or other symmetric solid body), by symmetry, the horizontal component of the resultant of forces on the body is null, and the vertical component is directed upward and is named as buoyant force of the fluid on the body and this phenomenon constitutes the Archimedes' principle (287-212 B.C.) [1 5]. For a more general case of a body with an arbitrary shape we can also mathematically demonstrate that the horizontal component of the resultant is null, remaining only the vertical component 6 . Another case that should be take into account is the case where a body is fully submerged in a liquid with a face touching the walls of a container. In this case the 'buoyant' force is not necessarily upward, and if the body is at the bottom of the container (bottom case) the force is downward and increases linearly with depth, as demonstrated in reference [6] and experimentally shown by Lima et al. [7]. The Archimedes' principle allows us to understand how a ship constructed of metallic parts and that may weigh several tons floats in the water, or as balloons and dirigibles ascend in the air. Another application of Archimedes' principle concerns the functioning of a hydrometer, which is an instrument used for the measurement of the density of liquids 8. In this work, the Archimedes' principle plus the definition of density $[9]$ are used for a simple description of how containers found in daily life float or not in the water. The analysis is based on the relation among the ratios volume of air within the container to the total volume; volume of water within the container to the total volume; volume of container walls to the total

*Correspondence email address: edup2112@gmail.com volume, and on the fraction of the total volume that is submersed. And then, after the theoretical description, a simple experimental demonstration on floating bodies is proposed.

\section{The body floats (or not) in the water}

Let us suppose that a body floating in the water be a closed container with its interior filled with a part of air and a part of water. Therefore, we can write that its total volume is the sum of the volumes of air and water inside it with the volume of its walls,

$$
V_{\text {cont }}=V_{\text {air }}+V_{\text {water }}+V_{\text {walls }} .
$$

Let us consider that the air volume occupies a fraction $f_{1}$ of the total volume of the container, the volume of water occupies a fraction $f_{2}$ of the total volume, and its walls occupy a fraction $f_{3}$ of the total volume. Thus, we can write that

$$
f_{1}=\frac{V_{\text {air }}}{V_{\text {cont }}}, \quad f_{2}=\frac{V_{\text {water }}}{V_{\text {cont }}} \quad \text { and } \quad f_{3}=\frac{V_{\text {walls }}}{V_{\text {cont }}},
$$

and therefore

$$
f_{1}+f_{2}+f_{3}=1
$$

Note that by definition we have

$$
0 \leq f_{1}<1, \quad 0 \leq f_{2}<1 \quad \text { and } \quad 0<f_{3} \leq 1
$$

To say that the container floats on the water surface means that the sum of the vertical forces is null:

$$
E=P,
$$

where $P$ is the weight of the container, and $E$ is the buoyancy. 
The weight of a body is given by the product of its mass by the gravitational acceleration $g$,

$$
P=\left(m_{\text {air }}+m_{\text {water }}+m_{\text {walls }}\right) g,
$$

where $m_{\text {air }}$ is the mass of air within the container, $m_{\text {water }}$ is the mass of water, and $m_{\text {walls }}$ is the mass of its walls. According to Archimedes' principle, the buoyant force is an upward force that a body immersed in a fluid experiences, and is given by the weight of the fluid displaced by the body [3]. The volume of displaced fluid (water in this case) corresponds to the volume of the container that is submerged ${ }^{1}$ Thus,

$$
E=\text { (mass of displaced fluid) } g \text {. }
$$

Recalling that the density of a body $(\rho)$ is defined as the ratio between its mass and its volume ${ }^{2}$

$$
\rho_{\text {water }}=\frac{\text { mass of displaced water }\left(m_{\text {displaced }}\right)}{\text { volume of displaced water }\left(V_{\text {displaced }}\right)},
$$

or

$$
m_{\text {displaced }}=\rho_{\text {water }} \cdot V_{\text {displaced }}=\rho_{\text {water }} \cdot f V_{\text {cont }},
$$

considering that a fraction $f$ of the volume of the container is submersed. Note that by definition

$$
0<f \leq 1
$$

So, the buoyant force can be written

$$
E=\rho_{\text {water }} \cdot f . V_{\text {cont }} \cdot g .
$$

Inserting Eqs. (6) and (10) in (5) results

$$
m_{\text {air }}+m_{\text {water }}+m_{\text {walls }}=\rho_{\text {water }} f V_{\text {cont }},
$$

and expressing the masses as a function of densities and volumes we obtain

$$
\rho_{\text {air }} \cdot V_{\text {air }}+\rho_{\text {water }} \cdot V_{\text {water }}+\rho_{\text {walls }} \cdot V_{\text {walls }}=\rho_{\text {water }} \cdot f V_{\text {cont }}
$$

or yet, replacing the ratios given in 2 and solving to $f$ we have

$$
f=\frac{\rho_{\text {air }}}{\rho_{\text {water }}} f_{1}+f_{2}+\frac{\rho_{\text {walls }}}{\rho_{\text {water }}} f_{3},
$$

Or

$$
f=A f_{1}+f_{2}+B f_{3},
$$

${ }^{1}$ When a body floats in the water it is also partially submerged in the air, and therefore the upthrust due to air must be considered. Nevertheless, as the density of air is much smaller than the density of water, we will neglect this contribution to the total upthrust.

${ }^{2}$ The concept of density applies to an object or to a substance. Thus, to a glass container filled with water we can define the density of the object (glass plus water), the density of the glass and the density of the water. where

$$
A=\frac{\rho_{\text {air }}}{\rho_{\text {water }}} \quad \text { and } \quad B=\frac{\rho_{\text {walls }}}{\rho_{\text {water }}}
$$

are the densities of the air and the material constituting the container walls relative to the density of the water.

Finally, considering Eq. 12 and more the fact that $f_{1}+f_{2}+f_{3}=1$ we get

$$
f_{1}=\frac{1-f-(1-B) f_{3}}{1-A}
$$

and

$$
f_{2}=\frac{-A+f+(A-B) f_{3}}{1-A}
$$

respectively to the ratios air volume/container volume and water volume/con-tainer volume as a function of the densities of air and material which forms the container walls relative to the density of the water, and also as a function of the fraction of the submersed container volume.

To say that the container sinks means that

$$
P>E \text {. }
$$

Now, provided that the container is fully submersed, the volume of displaced water is equal to the volume of the container and in this case we have

$$
m_{\text {air }}+m_{\text {water }}+m_{\text {walls }}>\rho_{\text {water }} \cdot V_{\text {cont }},
$$

or

$$
\rho_{\text {air }} \cdot V_{\text {air }}+\rho_{\text {water }} \cdot V_{\text {water }}+\rho_{\text {walls }} \cdot V_{\text {walls }}>\rho_{\text {water }} \cdot V_{\text {cont }}
$$

or yet, with the ratios given in $(2)$ and $(13)$ we have

$$
A f_{1}+f_{2}+B f_{3}>1
$$

or

$$
f^{\prime}>1, \quad \text { with } f^{\prime}=A f_{1}+f_{2}+B f_{3} .
$$

We can note that the expression to $f^{\prime}$ is the same as the expression to $f$ (Eq. (12)). Thus, calling indiscriminately $f$ and $f^{\prime}$ of $F$ we have

$$
0<F \leq 1, \quad \text { the container floats, }
$$

and

$$
F>1, \quad \text { the container sinks. }
$$

At standard sea-level atmospheric pressure and ambient temperature the density of water is $\rho_{\text {water }}=0.9982 \mathrm{~g} / \mathrm{cm}^{3}$ and the density of air is $\rho_{\text {air }}=0.001204 \mathrm{~g} / \mathrm{cm}^{3}$ |10. Then, the $A$ parameter is very well established and is equal to 0.001206; and the parameter $B$ depends on the material from which the walls of the container are formed.

In the following we will analyze four general cases. 
1. The container is filled with air and water in a certain proportion, $f_{1} \neq 0$ and $f_{2} \neq 0$. Let us then call the ratio $f_{1} / f_{2}$ of $r$. With this, equations (14) and 15 can be combined to eliminate $f_{2}$ :

$$
F=\frac{r A+1+f_{3}(-r A-1+B+r B)}{1+r} .
$$

The above equation tells us that $F$ will be independent of the value of $f_{3}$ to

$$
-r A-1+B+r B=0,
$$

or

$$
r=\frac{1-B}{B-A} .
$$

And the corresponding value of $F$ is

$$
F=\frac{r A+1}{1+r}=\frac{\left(\frac{1-B}{B-A}\right) A+1}{1+\left(\frac{1-B}{B-A}\right)}=B .
$$

The above expressions indicate that there is a value of the ratio $f_{1} / f_{2}$ which always gives the same value of the quantity $F$ for any value of the fraction of the volume of the container walls relative to the total volume, $f_{3}$. Furthermore, as the ratio $f_{1} / f_{2}$ is by definition a positive number it means that this result is only valid for containers whose wall materials have densities smaller than that of water, that is, $B<1$ (see Eq. (24) and therefore the container always floats. Otherwise, for $B>1$, we obtain from 22 that the container only floats to

$$
\begin{aligned}
& r \geq \frac{(1-B) f_{3}}{A-1+(B-A) f_{3}} \\
& \text { and } \\
& f_{3} \leq \frac{r(1-A)}{r(B-A)+B-1}
\end{aligned}
$$

2. The container is completely filled with water, $f_{1}=$ 0 .

From Eq. 14 we can write

$$
1-F-(1-B) f_{3}=0
$$

or

$$
F=1-(1-B) f_{3}
$$

for $B>1$ (glass, for example) $1-B$ is always negative, we see that $F>1$ which means that the container always sinks.

for $B<1$ (polyethylene, for example) $1-B$ is always positive, we see that $F<1$ which means that the container always floats.
3. The container is completely filled with air, $f_{2}=0$. From Eq. 15 we can write

$$
-A+F+(A-B) f_{3}=0
$$

or

$$
F=A-(A-B) f_{3} .
$$

Since $A$ is always smaller than 1 and $B<1$ or $B>1$, the value of $F$ depends on the value of $f_{3}$, that is, the thicknesses of the walls of the container. The body sinks to

$$
f_{3}>\frac{A-1}{A-B},
$$

and floats to

$$
f_{3} \leq \frac{A-1}{A-B} .
$$

4. The container is a solid block, with no air and no water inside it, $f_{1}=f_{2}=0$ and $f_{3}=1$.

We can see directly from Eqs. (12), 14 or 15 that

$$
F=B \text {, }
$$

which corresponds to the expected general case, that is, the body sinks to

$$
B>1
$$

and floats to

$$
B \leq 1
$$

In Fig. 1 is shown as the quantity $F$ varies as a function of the ratio air volume/water volume $\left(r=f_{1} / f_{2}\right)$ for containers of walls formed by polypropylene $(\rho=0.92$ $\left.\mathrm{g} / \mathrm{cm}^{3}\right)$ and common glass $\left(\rho=2.6 \mathrm{~g} / \mathrm{cm}^{3}\right)$, and at fixed values of fraction of the volume of the walls in relation to the total volume, $f_{3}$. For polypropylene it can be observed that the container always floats $(F<1)$ to any proportion between the volume of air and the volume of water. It can be also observed that this buoyant force is larger (smaller values of $F$ ), as smaller is the fraction of the volume of the walls in relation to the total volume $\left(f_{3}\right)$, and that is a value of the ratio $f_{1} / f_{2}$ that always provides the same value of the quantity $F$ for any value of $f_{3}$ as the above case $i$. For the glass it can be seen that the container always floats only for values of ratio between the volume of the walls and the total volume $\left(f_{3}\right)$ smaller than 0.01 (containers with very thin walls), and always sinks to values of $f_{3}$ greater than 0.39 . At $f_{3}=0.2$ the container sinks up to a certain value of $r$ and then starts to float. Whether or not the container floats depends on the values of $f_{3}$ and $r$, according to the limits given by expressions (26). Another behavior that can be noticed is that for both materials the quantity $F$ tends to the value of the density of the wall material relative to the density of the water, as the container becomes more and more massive $\left(f_{3} \rightarrow 1\right)$. 


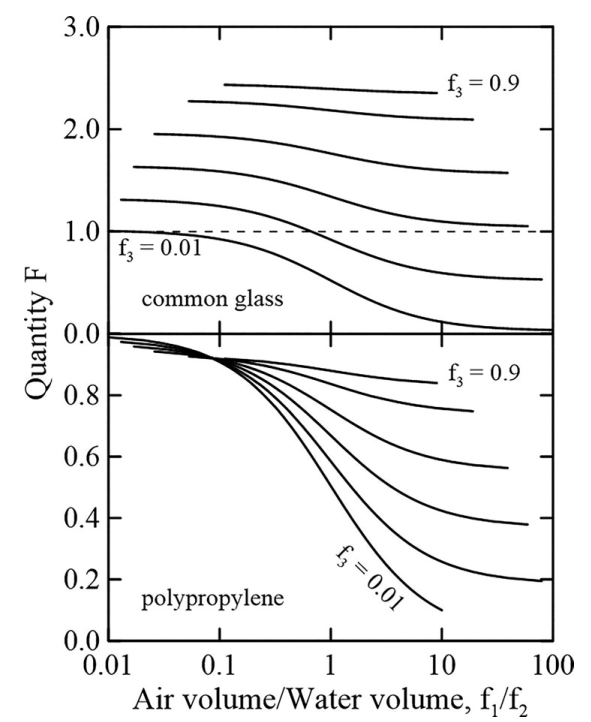

Figure 1: The quantity $F$ as a function of the ratio volume of air/volume of water in the container. Curves are shown for values of $f_{3}$ equal to $0.01,0.2,0.4,0.6,0.8$ and 0.9 . Bottom, polypropylene; top, common glass.

In Fig. 2 is shown as the quantity $F$ varies as a function of the ratio volume of walls/volume of container $\left(f_{3}\right)$ for containers of walls formed of polypropylene and common glass, and at fixed values of the ratio air volume/water volume $\left(f_{1} / f_{2}\right)$. Once again, it can be seen that the container formed of polypropylene always floats $(F<1)$ to any proportion between the volume of air and water and for any fraction of the volume of the walls in relation to the total volume, and this buoyant force is greater at greater values of the proportion of air in relation to water within the container. For the glass the container always sinks to values of the ratio air volume/water volume smaller than 0.02 ; at greater $r$ the container floats or not according to the limits given in (26). Figure 2 also shows a linear behavior of the quantity $F$ with the ratio of the volume of the walls to the total volume of the container for both polypropylene and glass, and for both materials the quantity $F$ tends to the value of the density of the wall material relative to the density of the water, according to Eq. (29), as the container becomes more and more massive $\left(f_{3} \rightarrow 1\right)$.

\section{The connexion with experiment $-\mathrm{A}$ simple demonstration}

In everyday life we find containers mainly made of aluminium, glass and plastics (cans and bottles of soft drinks and food, bottles of medicines and supplements, etc.). Among the plastic containers the most common are formed of polyethylene terephthalate (PET), high density polyethylene (HDPE), low density polyethylene (LDPE), poly(vinyl) chloride (PVC), polypropylene (PP) and polystyrene (PS). Plastic containers can generally be identified by observing the existence of a triangle of

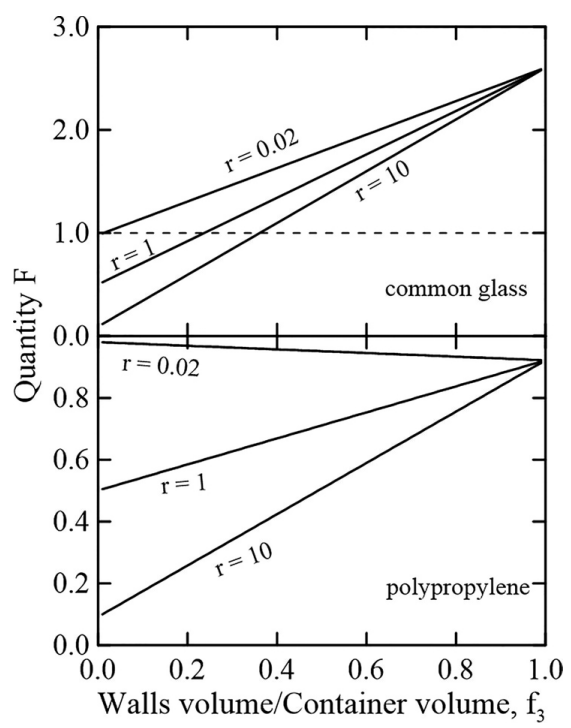

Figure 2: The quantity $F$ as a function of the ratio volume of the walls/volume of the container. Curves are shown for values of $r=f_{1} / f_{2}$ equal to $0.02,1$ and 10 . Bottom, polypropylene; top, common glass.

rounded vertices with a number inside it, as shown in Tab. 1 .

A basic demonstration of the principles described in section 2 consists in to obtain some containers formed by the materials listed in Tab. 1 and proceed as follows:

- We completely fill an aluminium can, a glass bottle or PET container with water and drop it on the surface of the water (from a box or a deep enough bucket). It can be seen that the they sink completely into the water, as the case 2 described in page $3(\rho>1, B>1, F>1)$.

- We completely fill a container of HDPE or polypropylene with water and drop it onto the surface of the water. It can be seen that both containers float in the water, according to the case 2 described in page $3(\rho<1, B<1, F<1)$.

- We put an empty aluminium can (i.e., filled only with air) of very thin walls on the surface of the water. It can be seen that the can floats in the water, as described in the case $3\left(f_{3}<\frac{A-1}{A-B}\right)$.

- We put an empty container made of polypropylene (or HDPE or LDPE) on the surface of the water. It can be seen that the container always floats regardless of the thickness of its walls, provided that $\frac{A-1}{A-B}$ is always greater than 1 .

- We put an empty glass bottle on the surface of the water. As in the previous case we observe that the bottle floats in the water if its walls are very thin, according to the case 3 described in page 3 $\left(f_{3}<\frac{A-1}{A-B}\right)$.

- Now we place an empty glass container with very thick walls (some perfume glasses have this characteristic). We observe in this case that the container 
Table 1: Densities of some materials commonly used in the production of containers [10].

\begin{tabular}{lccc}
\hline Name & Symbol/Acronym & Code & $\begin{array}{c}\text { density } \\
\left(\mathrm{g} / \mathrm{cm}^{3}\right)\end{array}$ \\
\hline Steel & - & - & 7.8 \\
Aluminium & $\mathrm{Al}$ & - & 2.7 \\
Common glass & - & - & $2.4-2.8$ \\
Polyethylene terephthalate & $\mathrm{PET}$ & 1 & $1.3-1.4$ \\
High Density Polyethylene & $\mathrm{HDPE}$ & 2 & $0.95-0.97$ \\
Poly(vinyl) Chloride & $\mathrm{PVC}$ & 3 & $1.39-1.42$ \\
Low Density Polyethylene & LDPE & 4 & $0.92-0.93$ \\
Polypropylene & $\mathrm{PP}$ & 5 & $0.91-0.94$ \\
Polystyrene & $\mathrm{PS}$ & 6 & $1.06-1.12$ \\
\hline
\end{tabular}

sinks, since for the glass it is reached the condition $f_{3}>\frac{A-1}{A-B}=0.384$, according to case 3 described in page 3

- We put solid blocks made of glass and polypropylene on the water. The block of glass sinks and the block of polypropylene floats according to case 4 .

- A last demonstration consists in to take an empty container formed of a material having a density greater than 1 and that floats on water. We gradually add water into the container, that is, we reduce the ratio $f_{1} / f_{2}$, until the container sinks. This behavior can be seen at the top of Figs. 11 (from right to left) and 2 (from bottom to top).

\section{Final remarks}

The Archimedes' principle, the concept of force equilibrium, and the definition of density are used for a simple description of the phenomenon of body floatation in the water. The analysis is carried out for containers commonly found in daily life and is based on the proportion among the volume of air inside the container, the volume of water inside it and the volume of the material of its walls, and also on the fraction of the total volume of the container that is submersed. Then, simple experimental demonstrations of easy performing and very low cost are proposed.

\section{Acknowledgement}

The author would like to thank the reviewer for his valuable comments and suggestions.

\section{References}

[1] R. Resnick, D. Halliday and J. Walker, Fundamentals of Physics (Wiley, New York, 2013), 10 $0^{\mathrm{a}}$ ed.

[2] V.C. Barbosa and A.M.S. Breitschaft, Revista Brasileira de Ensino de Física 28, 115 (2006).

[3] C. Gianino, Physics Education 42, 185 (2007).

[4] M. Kires, Physics Education 42, 484 (2007).

[5] M. Yadav, Physics Education 49, 523 (2014).

[6] F.M.S. Lima, European Journal of Physics 33, 101 (2012).
[7] F.M.S. Lima, G.M. Venceslau and G.T. Brasil, Revista Brasileira de Ensino de Física 36, 2309 (2014).

[8] B.M. Oliveira, J.M.M. Filho and J.C. Afonso, Revista Brasileira de Ensino de Física 35, 1601 (2013).

[9] S.J. Hawkes, Journal of Chemical Education 81, 14 (2004).

[10] D.R. Lide, CRC Handbook of Chemistry and Physics (CRC Press, Boca Raton, 2005). 